Article

\title{
Hedgehog Pathway Blockade Inhibits Melanoma Cell Growth in Vitro and in Vivo
}

Kathryn E. O’Reilly ${ }^{1}$, Eleazar Vega-Saenz de Miera ${ }^{1}$, Miguel F. Segura ${ }^{2,3}$, Erica Friedman ${ }^{4}$, Laura Poliseno ${ }^{5}$, Sung Won Han ${ }^{6}$, Judy Zhong ${ }^{6}$, Jiri Zavadil ${ }^{2,7,8}$, Anna Pavlick ${ }^{9}$, Eva Hernando ${ }^{2}$ and Iman Osman ${ }^{1,9, *}$

1 Department of Dermatology, New York University School of Medicine, New York, NY 10016, USA; E-Mails: koreilly6@gmail.com (K.E.O.); Eleazar.Vega-SaenzdeMiera@nyumc.org (E.V.-s.M.)

2 Department of Pathology, New York University School of Medicine, New York, NY 10016, USA; E-Mails: Miguel.segura@vhir.org (M.F.S.); ZavadilJ@iarc.fr (J.Z.);

Eva.Hernando-Monge@nyumc.org (E.H.)

3 Laboratory of Translational Research in Childhood Cancer, Vall d'Hebron Research Institute, Barcelona 08035, Spain

4 Department of Surgery, New York University School of Medicine, New York, NY 10016, USA; E-Mail: efriedman324@gmail.com

5 Oncogenomics Unit, Core Research Laboratory, Istituto Toscano Tumori, Pisa 56124, Italy; E-Mail: Laura.poliseno@gmail.com

6 Department of Population Health, New York University School of Medicine, New York, NY 10016, USA; E-Mails: sungwonhan2@gmail.com (S.W.H.); Judy.Zhong@nyumc.org (J.Z.)

7 NYU Center for Health Informatics and Bioinformatics, New York University Langone Medical Center, New York, NY 10016, USA

8 International Agency for Research on Cancer, 69372 Lyon Cedex 08, France

9 Department of Medicine, New York University School of Medicine, New York, NY 10016, USA; E-Mail: Anna.Pavlick@nyumc.org

* Author to whom correspondence should be addressed; E-Mail: Iman.Osman@nyumc.org; Tel.: +1-212-263-9076; Fax: +1-212-263-9090.

Received: 3 September 2013; in revised form: 24 October 2013 / Accepted: 31 October 2013 / Published: 11 November 2013

Abstract: Previous reports have demonstrated a role for hedgehog signaling in melanoma progression, prompting us to explore the therapeutic benefit of targeting this pathway in melanoma. We profiled a panel of human melanoma cell lines and control melanocytes for altered expression of hedgehog pathway members and determined the consequences of both 
genetic and pharmacological inhibition of the hedgehog pathway activator Smoothened (SMO) in melanoma, both in vitro and in vivo. We also examined the relationship between altered expression of hedgehog pathway mediators and survival in a well-characterized cohort of metastatic melanoma patients with prospectively collected follow up information. Studies revealed that over $40 \%$ of the melanoma cell lines examined harbored significantly elevated levels of the hedgehog pathway mediators SMO, GLI2, and PTCH1 compared to melanocytes $(p<0.05)$. SMO inhibition using siRNA and the small molecule inhibitor, NVP-LDE-225, suppressed melanoma growth in vitro, particularly in those cell lines with moderate SMO and GLI2 expression. NVP-LDE-225 also induced apoptosis in vitro and inhibited melanoma growth in a xenograft model. Gene expression data also revealed evidence of compensatory up-regulation of two other developmental pathways, Notch and WNT, in response to hedgehog pathway inhibition. Pharmacological and genetic SMO inhibition also downregulated genes involved in human embryonic stem cell pluripotency. Finally, increased SMO expression and decreased expression of the hedgehog pathway repressor GLI3 correlated with shorter post recurrence survival in metastatic melanoma patients. Our data demonstrate that hedgehog pathway inhibition might be a promising targeted therapy in appropriately selected metastatic melanoma patients.

Keywords: melanoma; hedgehog; Smoothened; GLI2; PTCH1

\section{Introduction}

Melanoma incidence is quickly rising, with an estimated lifetime risk of 1 in 50 [1-3]. The average survival for a patient with metastatic disease is currently only 7 months $[3,4]$. Targeted therapies, such as B-RAF mutant kinase inhibitors, have shown dramatic initial responses in clinical trials, but resistance to single agent therapy inevitably ensues [5,6]. The CTLA-4 inhibitor, ipilimumab, induces more durable responses, but in just $20 \%$ of metastatic melanoma patients, and biomarkers for predicting response to this targeted agent are currently lacking [7,8]. Thus, despite recent advances, melanoma remains a clinical dilemma of considerable magnitude. We hypothesized that aberrantly activated developmental pathways may play an important role in melanoma. In this regard, several groups, including ours, have implicated the Notch pathway in melanoma progression $[9,10]$. Notch inhibitors currently in clinical trials have, however, shown limited efficacy in melanoma and gastrointestinal side effects remain an obstacle in it is clinical development [11-13].

Over the past five years, three independent groups have described the importance of yet another embryonic signaling cascade, the hedgehog pathway, in melanoma progression [14-16]. The hedgehog pathway is crucial in the embryo, ensuring proper spatiotemporal development. Physiologic hedgehog pathway activation occurs when secreted hedgehog ligand $(\mathrm{HH})$ binds to the membrane associated Patched 1 (PTCH1) receptor, leading to activation of the Smoothened G-protein coupled receptor-like receptor (SMO) [17]. SMO signaling activates the GLI family zinc finger (GLI) transcription factors and results in transcription of target genes which include mediators of the pathway such as GLI1, PTCH1, cyclins D\&E, and insulin-like growth factor binding proteins. While GLI1 and GLI2 mediate 
transcription of hedgehog target genes, GLI3 is a transcriptional repressor, leading to downregulation of hedgehog target genes. Aberrant activation of the pathway in adult tissue has been implicated in the pathogenesis of several cancers, and hedgehog signaling has been reported as a cancer stem cell pathway, mediating epithelial-to-mesenchymal transition via downregulation of E-cadherin [18-24]. Although no mutations in the hedgehog pathway have been described in melanoma, previous studies have demonstrated that hedgehog signaling is required for melanoma growth and metastasis in transgenic and xenograft mouse models [14,15]. Two studies also described high levels of GLI1 and GLI2 transcription factor mRNA in human metastatic melanoma tissue as compared to primary melanoma tissue $[15,16]$. Hedgehog pathway activity has also been shown to be necessary for the self-renewal of melanoma-initiating cells in vitro [25]. Most recently, the SMO antagonist NVP-LDE-225, (Novartis Pharma, AG, Basel, Switzerland) has been shown to inhibit melanoma growth both in vitro and in vivo [26]. These studies do not, however, examine the association between hedgehog pathway activity in melanoma and patient survival. In our study, we show that NVP-LDE-225, an oral hedgehog pathway inhibitor currently in a Phase II clinical trial, inhibits melanoma cell growth in vitro and in vivo, and we report, for the first time, the association of aberrant hedgehog pathway activity with decreased survival in melanoma patients.

\section{Experimental}

\subsection{Human Melanoma Cell Lines}

Primary melanoma lines WM35, WM1552c, WM1575, WM98.1, WM115, WM278, WM-793, WM-853, WM-902, WM-983, WM-1366, WM1361A, and WM3248 were purchased from the Wistar Institute (Philadelphia, PA, USA) and cultured in Mel 2\% medium [27]. SkMel29, SkMe185, SkMe194, SkMel100, SkMel103, SkMel147, SkMel173, SkMel187, SkMel192, SkMel197 were kindly provided by Dr. Alan Houghton (MSKCC), and A375, Lu451, and 501 Mel were purchased from ATCC and cultured in DMEM supplemented with 10\%FBS and 1\% penicillin/streptomycin.

\subsection{Data Mining of mRNA Microarray of Melanoma Cell Lines and qRT-PCR Confirmation}

The U133A 2.0 Affymetrix array was performed on four melanocyte controls (two normal human melanocytes and two immortalized melanocytes), four primary and 14 metastatic cell lines as described in reference [28]. A curated gene signature of hedgehog pathway activation containing 77 distinct genes involved in hedgehog signaling was generated with the Broad Institute Molecular Signature Database and gene expression of these markers was analyzed in each of the 22 melanoma lines (including four primary and 14 metastatic melanoma lines) and melanocytes using Genespring GX software (Agilent, Santa Clara, CA, USA). Hierarchical clustering generated with Genespring GX software was used to create a heat map of hedgehog pathway gene expression. Increased levels of hedgehog pathway members were confirmed via quantitative real time PCR (qRT-PCR). RT-PCR was performed using qScript cDNA Supermix (Quanta Biosciences, Gaithersburg, MD, USA) and qPCR performed with Absolute Blue qPCR Sybr Green Mix (Thermo Fisher Scientific, Waltham, MA, USA). qPCR data analysis was performed using the ABI 7900HT (Applied Biosystems, Carlsbad, CA, 
USA) sequence detection instrument and threshold cycle values converted to relative transcript levels using the comparative Ct Method.

\subsection{Data Mining of mRNA Microarray of Melanoma Cell Lines and qRT-PCR Confirmation, $m R N A$} Microarray of Metastatic Melanoma Specimens

Using the previously published Human Genome U133A 2.0 Affymetrix expression profile of 44 metastatic melanoma samples [29] from patients followed clinically for a median of 35.4 months as well as the Human Genome U133 Plus 2.0 expression profile of 30 additional metastatic melanoma samples that we recently generated, we examined whether the canonical hedgehog pathway mediators SHH, GLI1, GLI2, GLI3, SMO, or PTCH1 were associated with significantly decreased post-recurrence survival. RNA was isolated from the 30 additional metastatic melanoma specimens using the RNAeasy Kit (Qiagen Sciences, Germantown, MD, USA).The 30 additional metastatic melanoma patients were identified through the Interdisciplinary Melanoma Cooperative Group database at New York University School of Medicine (19 male, 11 female; median age, 63.4 years). Of the 30 specimens from 30 patients, 11 were lymph node metastases, 15 were skin metastases, and four were visceral metastases. The median follow-up time for the cohort from the time of primary diagnosis to last follow-up date was 70.08 months. The study was approved by the New York University Institutional Review Board, and all patients signed informed consent before enrollment. Relevant clinicopathologic, demographic, and survival data were recorded for all patients.

\subsection{Statistical Analysis}

Descriptive statistics were calculated for baseline demographic and clinicopathologic characteristics. Cox proportional hazards model dichotomized at the median expression value was used to examine the association between hedgehog pathway mediatory transcript levels and post-recurrence survival (time from first recurrence to death). Kaplan-Meier curves were generated using Graph Pad Prism 5.0 Software (LaJolla, CA, USA).

\subsection{In Vitro Cell Proliferation Assays of Cyclopamine, NVP-LDE-225, and Vemurafenib}

The indicated cell lines were seeded at a density of $1 \times 10^{4}$ cells per well in a 12-well dish in triplicate in DMEM medium. The day after (day 0), the medium was replaced, and DMSO, cyclopamine (Toronto Research Chemicals, North York, ON, Canada), the oral Smoothened inhibitor in Phase II clinical trials, NVP-LDE-225, (Novartis Pharma AG), or Vemurafenib (ChemieTek, Indianapolis, IN, USA) at indicated concentrations were added. At the indicated time points (3-12 days), cells were fixed in $10 \%$ formalin solution and stored in PBS at $4{ }^{\circ} \mathrm{C}$. After the final time point, all the plates were stained with crystal violet. After color elution with $10 \%$ acetic acid, optical density was read at $590 \mathrm{~nm}$. A representative curve of three independent experiments is reported.

\subsection{Cell Cycle and Apoptosis Analysis}

$5 \times 10^{5}$ A375 cells were plated in $10 \mathrm{~cm}$ plates, and $24 \mathrm{~h}$ later were treated with DMSO or $5 \mu \mathrm{M}$ NVP-LDE-225. After $72 \mathrm{~h}$ of treatment, cells were trypsinized, washed with PBS, and fixed in $70 \%$ 
ethanol. Prior to FACS analysis, fixed cells were stained with propidium iodide in PBS (25 $\mu \mathrm{g} / \mathrm{mL}$ ) containing $250 \mu \mathrm{g} / \mathrm{mL}$ RNase A. Pan-caspase activation and changes in mitochondrial potential were determined in A375 and WM3248 cells after $72 \mathrm{~h}$ of treatment with $10 \mu \mathrm{M}$ NVP-LDE-225 using the dual sensor MitoCasp ${ }^{\mathrm{TM}}$ assay (Cell Technology, Mountain View, CA, USA) according to the manufacturer's protocol. Stained cells were evaluated in an LSRII flow cytometer and analyzed using Flowing Software version 2.4 (Perttu Terho, Turku Centre for Biotechnology, Turku, Finland).

\section{7. siRNA Assays}

2-4 $\times 10^{4}$ melanoma cells were plated in antibiotic free medium into each well of a 12 well plate. $18 \mathrm{~h}$ later, 40 pmol of ON-Target plus SMPARTpool Human SMO (L-005726-00-0005), Human GLI1 (L-003896-00-0005), or Human GLI2 (L-006468-00-0005) siRNA (Thermo Scientific Dharmacon, Lafayette, CO, USA) diluted in Opti-Mem I reduced serum media were transfected into the cells using Lipofectamine 2000 (Invitrogen, Grand Island, NY, USA). The medium was changed after $6 \mathrm{~h}$ and cells were trypsinized for quantitative real time PCR or stained with crystal violet at indicated time points.

\subsection{Xenograft Assay of A375 Cells}

$1.5 \times 10^{6} \mathrm{~A} 375$ cells were injected into the flank of NOD/Scid/IL2 gamma receptor -/- (NOG) mice $(\mathrm{n}=20)$. Once tumors were palpable (6 days after injection), mice were randomized into two groups and vehicle $(\mathrm{n}=10)$ or NVP-LDE-225 was administered orally at $60 \mathrm{mg} / \mathrm{kg} / \mathrm{day}$. After one week of treatment, tumors were excised, weighed, and RNA was isolated from tumors for RT-PCR. Effective pathway inhibition was confirmed with qPCR of PTCH1 transcript levels in the tumors of NVP-LDE-225 treated mice as compared to vehicle treated mice.

\subsection{Pathway Analysis of SMO Inhibition Transcriptional Profile}

The transcriptional profile of NVP-LDE-225 and SMO siRNA treated WM3248 and A375 melanoma cells was generated using a Human Genome U133 Plus 2.0 genechip. The WM3248 and A375 cell lines were treated for $24 \mathrm{~h}$ with either $5 \mu \mathrm{M}$ NVP-LDE-225 or SMO siRNA. The significantly downregulated canonical signaling pathways in SMO inhibitor treated WM3248 and A375 cell lines, as compared to DMSO or non-targeting siRNA treated cells, respectively, were generated with Ingenuity Pathway Analysis software (Ingenuity Systems, Inc., Redwood City, CA). Canonical pathways analysis identified the pathways from the IPA library of canonical pathways that were most significant to the data set. Genes from the data set that met the fold change cutoff of 1.2 and were associated with a canonical pathway in the Ingenuity Knowledge Base were considered for the analysis. The significance of the association between the data set and the canonical pathway was measured in 2 ways: (1) A ratio of the number of molecules from the data set that map to the pathway divided by the total number of molecules that map to the canonical pathway is displayed. (2) Fisher's exact test was used to calculate a p-value determining the probability that the association between the genes in the dataset and the canonical pathway is explained by chance alone. 


\section{Results and Discussion}

\subsection{Expression of Hedgehog Pathway Members is Elevated in Melanoma Cell Lines compared to}

\section{Melanocytes}

We analyzed the expression of 77 genes from a curated gene signature of hedgehog pathway activation using our microarray expression profile of 18 melanoma cell lines and four melanocyte controls [28]. Array expression values of WNT5A and GLI2 were found to be significantly higher than melanocytes in over $50 \%$ of the melanoma cell lines, $(14 / 18$ and $14 / 18$ cell lines, respectively) (Figure 1$)(p<0.05)$.

Figure 1. Hedgehog pathway members are upregulated in a subset of melanoma cell lines as measured via affymetrix microarray analysis. SMO, GLI2, PTCH1, and WNT5a are upregulated, while negative regulators of hedgehog signaling such as protein kinase A (PRKA) and dual specificity tyrosine-phosphorylation-regulated kinase 2 (DYRK2) are downregulated as compared to melanocytes.

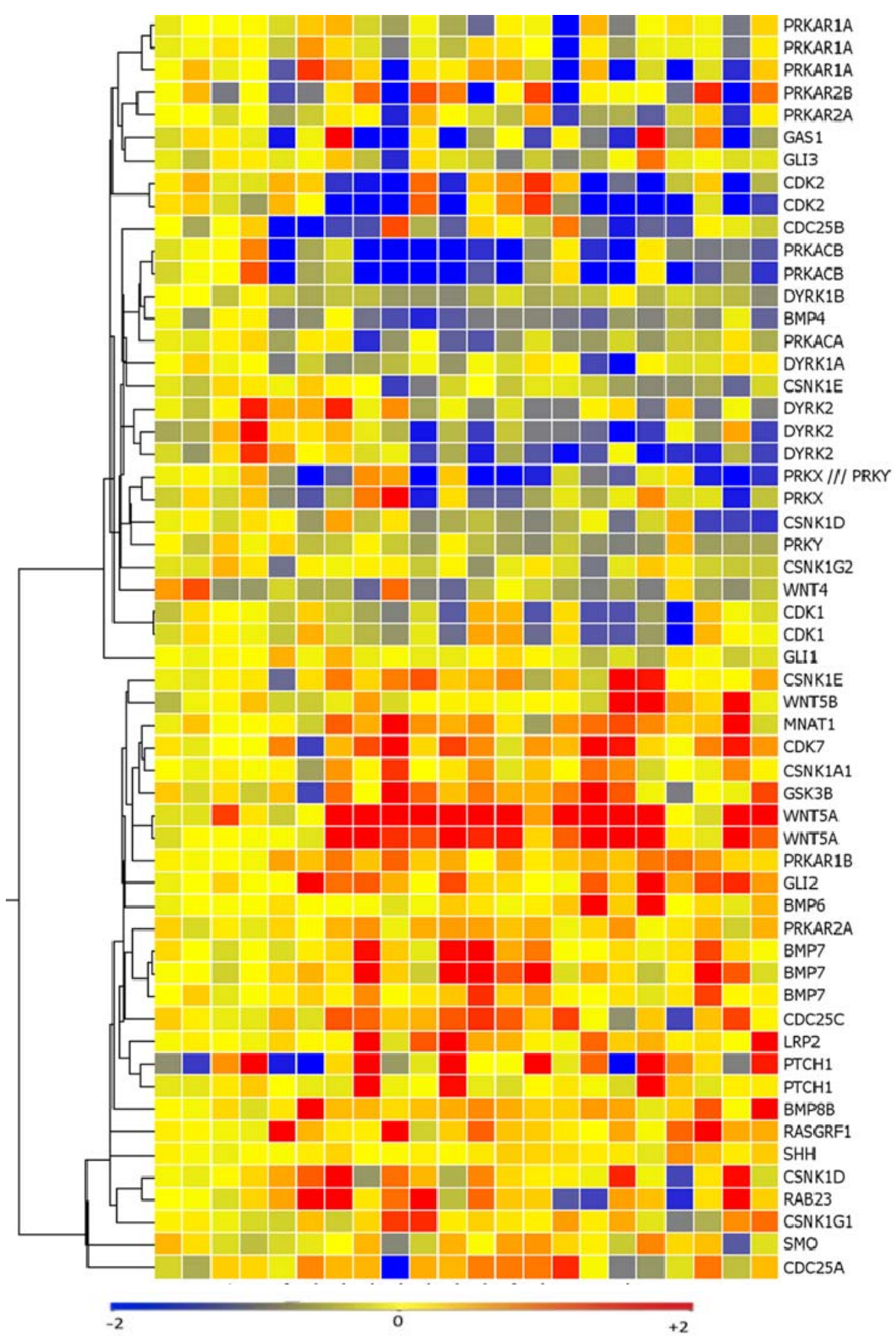


SMO and PTCH1 expression levels were also higher in a subset of melanoma cell lines as compared to melanocytes $(8 / 18$ and $8 / 18$ cell lines, respectively) $(p<0.05)$. There was also loss of expression of negative regulators of the hedgehog pathway such as protein kinase $A$ (PRKA) and dual-specificity tyrosine-(Y)-phosphorylation regulated kinase 2 (DYRK2) in a subset of melanoma cell lines $(13 / 18$ and 12/18 cell lines, respectively) $(p<0.05) .11$ cell lines expressed high levels of both WNT5a and GLI2 as compared to the melanocytes controls and 10/11 of these cell lines (91\%) displayed loss of both DYRK2 and PRKA (Figure 1). The elevated expression of PTCH1, SMO, GLI2, and WNT5A detected with array technology was validated in an expanded melanoma cell line panel of 26 melanoma cell lines using quantitative real time PCR (Figure $2 a-d$ ).

Figure 2. Hedgehog pathway members are upregulated in a subset of melanoma cell lines. (A-D) Elevated transcript levels of SMO, GLI2, PTCH1, and WNT5a detected by Affymetrix microarray analysis were confirmed by quantitative RT-PCR. Values represent the mean \pm standard deviation of triplicate qPCRs from three different experiments. Open bars represent melanocytes controls, while the closed bars represent melanoma cell lines. The cell lines beginning with "WM" are primary melanoma cell lines, while the cells lines beginning with "SK-MEL" and the A375, LU451, and 501MEL cell lines are metastatic melanoma cell lines. (*) Indicates that the cell line is a V600E B-RAF mutant.

A
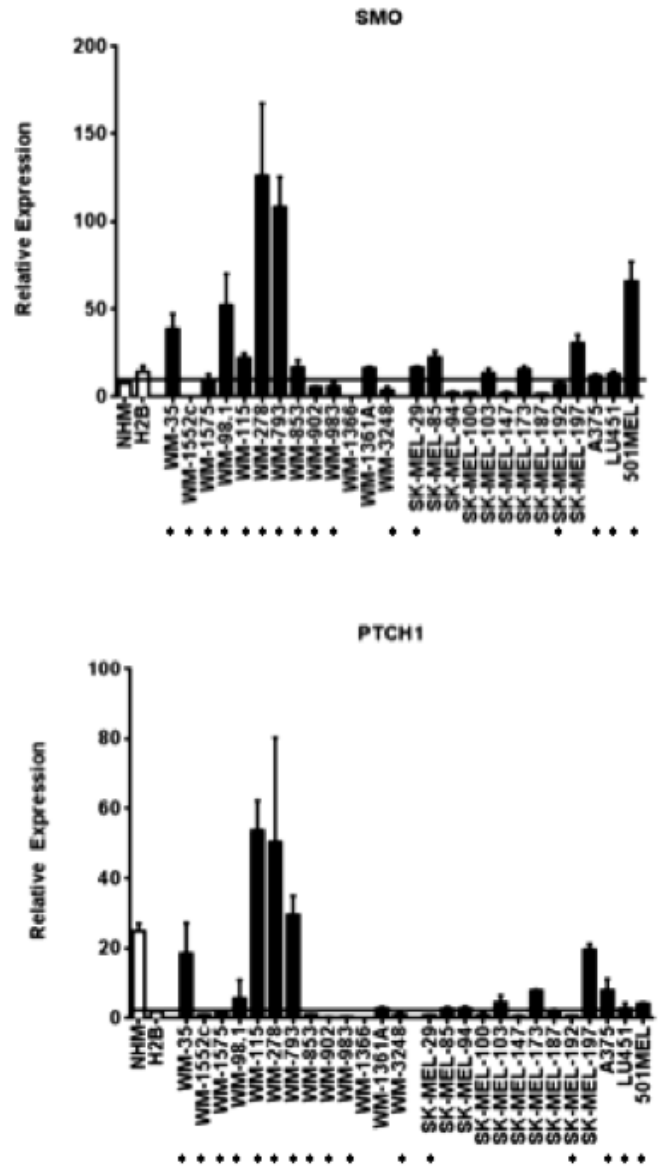

B
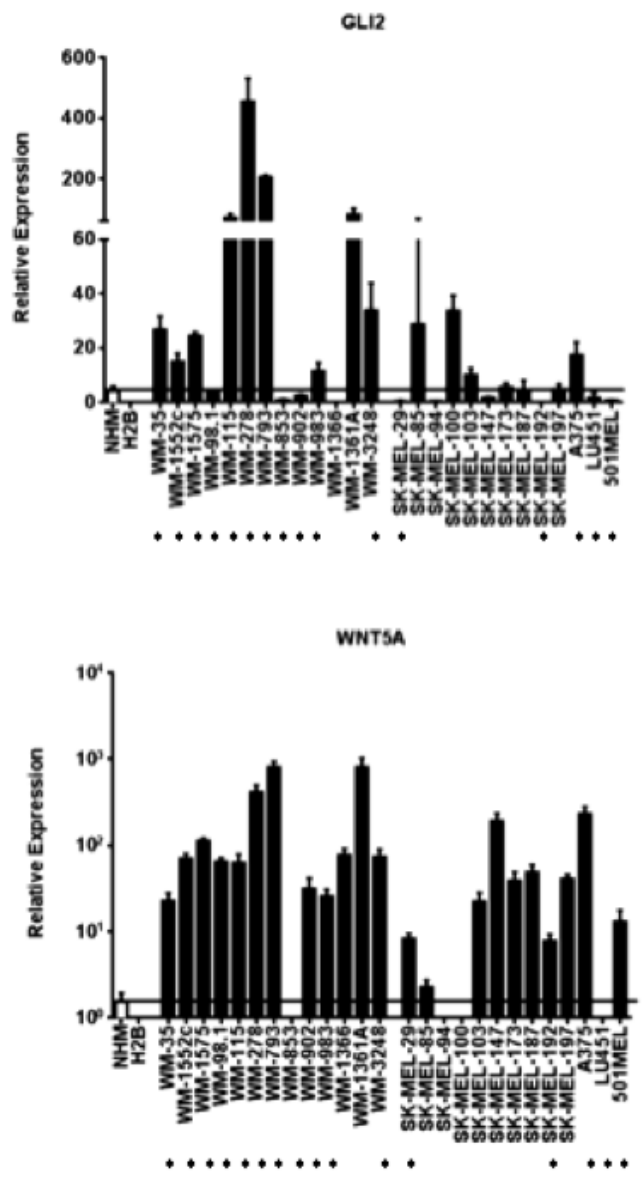

As compared to normal or immortalized melanocytes, GLI2 mRNA was found to be elevated in 13/26 (50\%) melanoma cell lines and SMO expression was elevated in 13/26 (50\%) cell lines. Six of 
the cell lines overexpressing GLI2 as compared to melanocytes concomitantly overexpressed SMO. The GLI2 transcriptional target WNT5A was found to upregulated in 22/26 (85\%) melanoma cell lines as compared to melanocyte controls. Finally, the hedgehog pathway transcriptional target PTCH1 was elevated in 10/26 (39\%) melanoma cell lines as compared to immortalized melanocytes. Of note, in our microarray analyses, GLI1 was not found upregulated in our panel of melanoma cells as compared to melanocytes. GLI1 was found to be expressed at much lower levels than GLI2, SMO, or PTCH1 mRNA as measured by quantitative qRT-PCR (Figure 3a). The increased expression of two effectors of hedgehog signaling, such as GLI2 and SMO, as well as transcriptional targets of hedgehog signaling like PTCH1 and WNT5A, suggests that half of the melanoma cell lines tested harbor upregulated hedgehog pathway activity as compared to control melanocytes.

\subsection{SMO Inhibition Decreases Melanoma Cell Proliferation in Vitro}

We then examined the effect of hedgehog pathway inhibition on melanoma growth. The small molecule SMO inhibitor cyclopamine decreased cell proliferation after $72 \mathrm{~h}$ of treatment in the A375 and WM3248 cell lines, which demonstrated modestly elevated GLI2, SMO, PTCH1 expression (between 10 and 60 fold increase as measured by qPCR) (Figure 3b). Cyclopamine demonstrated weaker antiproliferative effects in the SKMel94 cell line with low GLI2, SMO, PTCH1 expression ( $<5$ fold increase), and in the WM278 cell line, which harbors a one copy GLI2 and two copy SMO allele gain as detected by a DNA SNP array [26] and overexpresses GLI2 and SMO relative to melanocytes (fold increase for both GLI2 and SMO > 100) and the majority of other melanoma cell lines in our panel ( $\mathrm{IC}_{50}$ values of 47.5 and $91.9 \mu \mathrm{M}$, respectively) (Figure 3b). Interestingly, we observed that cell lines with modest expression of pathway members were more sensitive to SMO inhibition than those with either very low (less than 20 fold increase) or very high expression (over 150 fold increase) of GLI2, SMO, and PTCH1.

Figure 3. Melanoma cell lines harboring modest expression of GLI2 and SMO were more sensitive to cyclopamine as compared to those cell lines with overexpression or absence of GLI2 and SMO. (A) Baseline levels of GLI1, GLI2, SMO, and PTCH1 were measured by qRT-PCR in four melanoma cell lines. (B) The A375 and WM3248 cell lines were sensitive to cyclopamine $\left(\mathrm{IC}_{50}<10 \mu \mathrm{M}\right)$, whereas the WM278 and SkMe194 cell lines were resistant to cyclopamine $\left(\mathrm{IC}_{50}>45 \mu \mathrm{M}\right)$. In $\mathrm{A}$, values represent the mean \pm standard deviation from a representative experiment performed three times with similar results. In B the values represent the mean from a representative experiment performed three times with similar results.

A

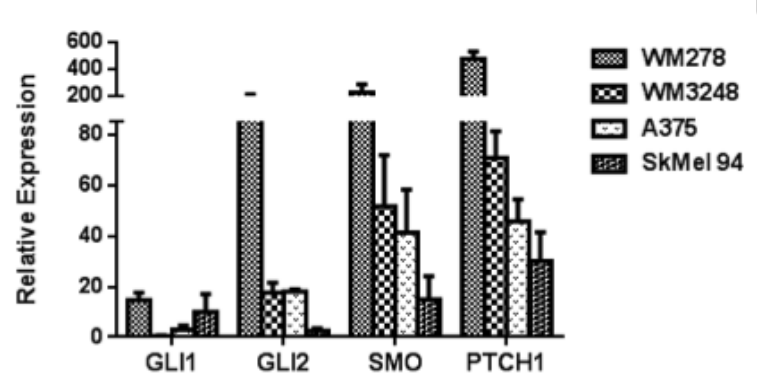

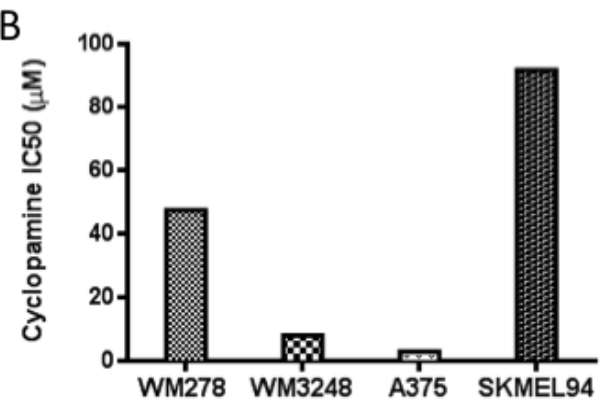


To account for potential off-target effects of small molecule SMO inhibition, we employed SMO siRNA to determine whether genetic interference with the hedgehog pathway would also inhibit growth. Transient transfection of siRNA against SMO demonstrated a similar antiproliferative effect at $72 \mathrm{~h}$ as three days of $5 \mu \mathrm{M}$ cyclopamine treatment in the WM3248 cell line (Figure 4a). siRNA to SMO inhibited proliferation in the WM3248 cell lines better than GLI1 or GLI2 silencing (Figure 4b). In the WM278 cell line, neither SMO nor GLI1 silencing inhibited proliferation as compared to non-targeting siRNA transfected cells (Figure $4 b$ ).

Figure 4. Smoothened inhibition has antiproliferative effects in melanoma in vitro. (A) siRNA against $S M O$ demonstrates a similar antiproliferative effect as $5 \mu \mathrm{M}$ cyclopamine after three days in the WM3248 cell line, as measured by crystal violet staining. (B) Knockdown of hedgehog pathway mediators using siRNA to target GLI1, GLI2, or SMO was performed in the WM3248 and WM278 cell lines. In the WM3248 cell line, which was sensitive to cyclopamine, siRNA to SMO has better antiproliferative effects than GLI1 or GLI2 inhibition. In the WM278 cell line, which is resistant to cyclopamine, and harbors amplification of SMO and GLI2, GLI2 inhibition decreases proliferation more than inhibition of either GLI1 or SMO (*,p<0.05). (C) The SMO inhibitor NVP-LDE-225 inhibits melanoma cell growth in the A375, WM278, WM3248, and SkMe194 cell lines. (D) $5 \mu \mathrm{M}$ NVP-LDE-225 inhibits hedgehog signaling in vitro as evidenced by decreased PTCH1 transcript levels after $24 \mathrm{~h}$ of treatment (*, $p<0.05)$. In A-C, values represent the mean \pm standard deviation from a representative experiment performed three times with similar results. In $\mathrm{D}$, the values represent the mean \pm standard deviation of triplicate qPCRs from three different experiments.

A
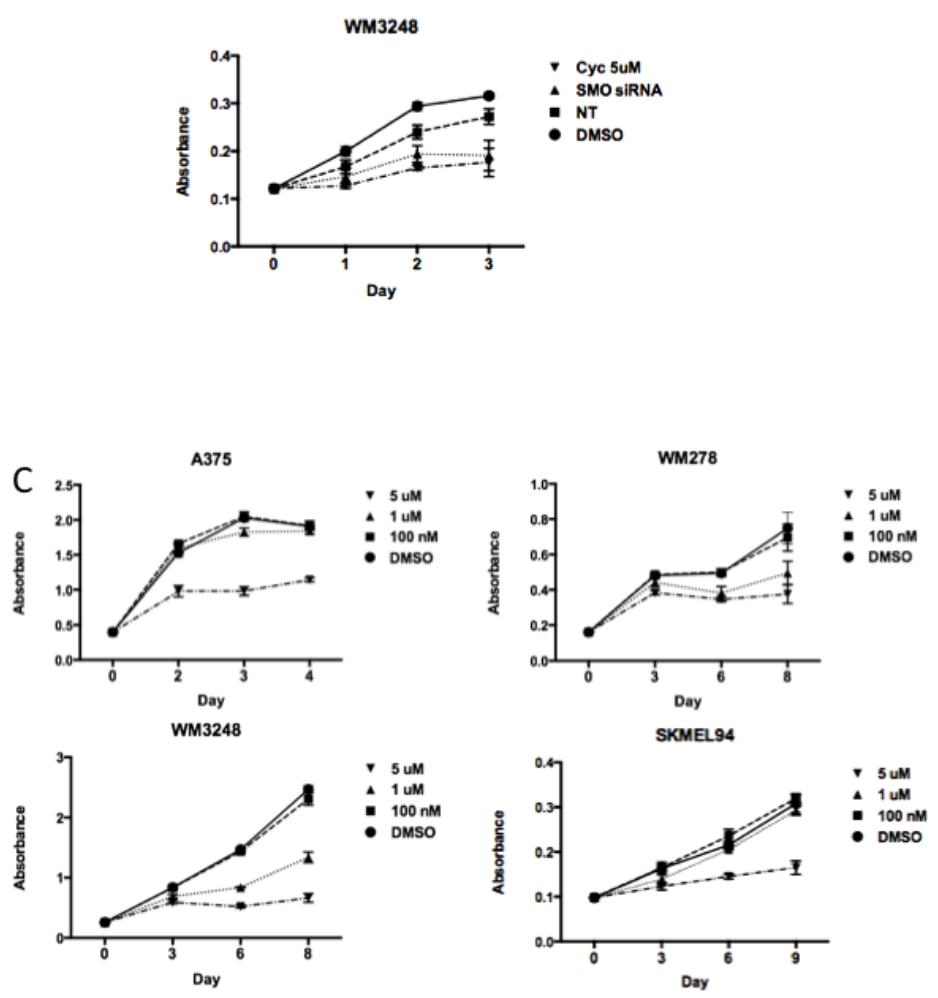

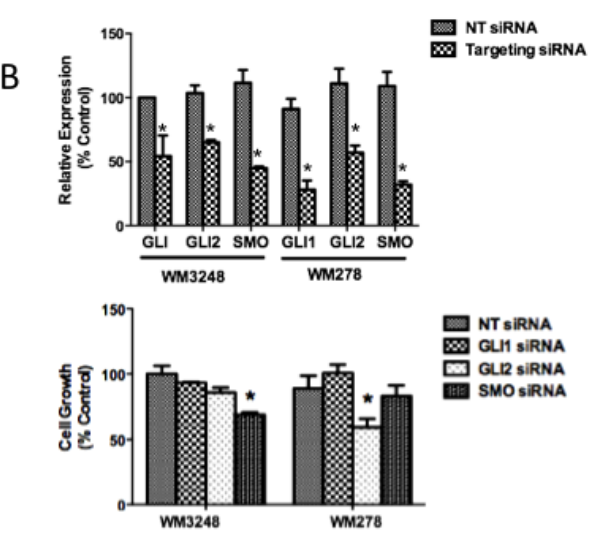

D

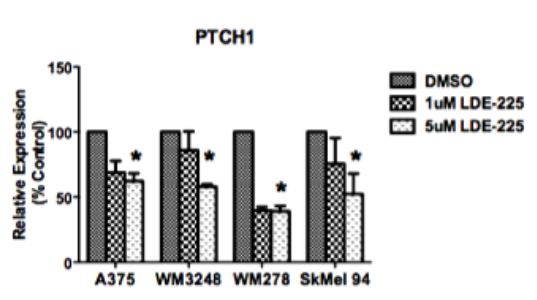


In WM278, GLI2 silencing inhibited proliferation better than inhibition of either SMO or GLI1. The weak antiproliferative effect of SMO silencing in the WM278 cell line suggests that overexpression of GLI2, a downstream hedgehog pathway effector, may render melanoma cells resistant to SMO inhibition. SMO silencing by siRNA mimicked the effect of cyclopamine in melanoma cells, with antiproliferative effects in cells that show an $\mathrm{IC}_{50}$ of less than $10 \mu \mathrm{M}$ and a lack of antiproliferative effect in cells that displayed an $\mathrm{IC}_{50}$ greater than $45 \mu \mathrm{M}$ (Figure $4 \mathrm{~b}$ ).

\subsection{NVP-LDE-225 Inhibits Melanoma Cell Proliferation and Induces Apoptosis in Vitro}

As cyclopamine's unfavorable pharmacokinetic properties preclude its use in humans, we investigated the effects of the clinically relevant SMO inhibitor, NVP-LDE-225, on melanoma cell proliferation. The small molecule NVP-LDE-225 is a well-tolerated oral SMO inhibitor currently in Phase II clinical trials in advanced basal cell carcinoma. We found that NVP-LDE-225 decreased melanoma cell proliferation both in vitro and in vivo. NVP-LDE-225 inhibited the proliferation of A375, WM3248, WM278, and SkMel 94 cell lines after 3-6 days of treatment (Figure 4c). NVP-LDE-225 effectively inhibited hedgehog pathway signaling, as indicated by a decrease in PTCH1 expression after $24 \mathrm{~h}$ of treatment in all four cell lines tested (Figure 4d).

As observed with cyclopamine treatment, cell lines with moderate expression of hedgehog pathway mediators GLI2 and SMO were more sensitive to NVP-LDE-225 than those cell lines harboring amplification and/or overexpression of hedgehog family members (Figure 5a). $\mathrm{IC}_{50} \mathrm{~S}$ for WM3248, WM35, WM1575, and A375 cell lines with moderate expression of both GLI2 and SMO ranged from 1.4-6.8 $\mu \mathrm{M}$, while $\mathrm{IC}_{50}$ s for WM793 and WM278 which overexpress GLI2 and SMO were over $50 \mu \mathrm{M}$. The SkMel 192 and SkMel 94 cell lines which lack expression of SMO and GLI2 were most resistant to NVP-LDE-225 with $\mathrm{IC}_{50} \mathrm{~S}$ of 81.38 and $139.3 \mu \mathrm{M}$, respectively (Figure 5a).

In order to ascertain how the inhibition of the hedegehog pathway impacts on cell number, we analyzed the cell cycle profile of melanoma cells sensitive to NVP-LDE-225. Cell cycle profiles revealed no major differences between NVP-LDE-225 treated A375 and WM3248 cells versus control (data not shown). However, cell death analysis using a pan-caspase detection assay and a mitochondrial potential assay revealed an increased percentage of apoptotic cells after $72 \mathrm{~h}$ of NVP-LDE-225 treatment in both the A375 and WM3248 cells (Figure 5b). As the four melanoma cell lines tested that were most sensitive to NVP-LDE-225 were all V600E B-RAF mutant cell lines (as confirmed by DNA sequencing in our laboratory), we examined a potential synergism between NVP-LDE-225 and the BRAF inhibitor vemurafenib. For that, A375 cells were treated with DMSO, NVP-LDE-225 (10 $\mu$ M), the B-RAF inhibitor vemurafenib $(100 \mathrm{nM})$, or the combination of both for three days. We found a $31 \%$ reduction in cell growth in the combination treated cells as compared to single agent treated cells $(p=0.0025)$ (Figure 5c). 
Figure 5. The SMO inhibitor NVP-LDE-225 inhibits proliferation in primary and metastatic melanoma cell lines in vitro. (A) Primary and metastatic melanoma cell lines demonstrate variable sensitivity to NVP-LDE-225. Cell lines harboring modest expression of GLI2 and SMO are more sensitive to NVP-LDE-225 ( IC $_{50}<15 \mu \mathrm{M}$ ) as compared to those cell lines with overexpression of GLI2 and SMO (WM278 and WM793) or lack of pathway activity (SkMel94 and SkMel100) $\left(\mathrm{IC}_{50}>50 \mu \mathrm{M}\right)$. (B) $10 \mu \mathrm{M}$ NVP-LDE-225 induces apoptosis in A375 and WM3248 cells as detected by an increase in pan-caspase positive cells and in cells with loss of mitochondrial membrane potential after $72 \mathrm{~h}$ of treatment. (C) Combination LDE-225 $(10 \mu \mathrm{M})$ and vemurafenib (100 nM) inhibits proliferation better than either single agent or vehicle after $72 \mathrm{~h}$ of treatment $(*, p<0.003)$. In $\mathrm{A}-\mathrm{B}$, values represent the mean from a representative experiment performed three times with similar results. In $\mathrm{C}$, values represent the mean \pm standard deviation from a representative experiment performed three times with similar results.

A

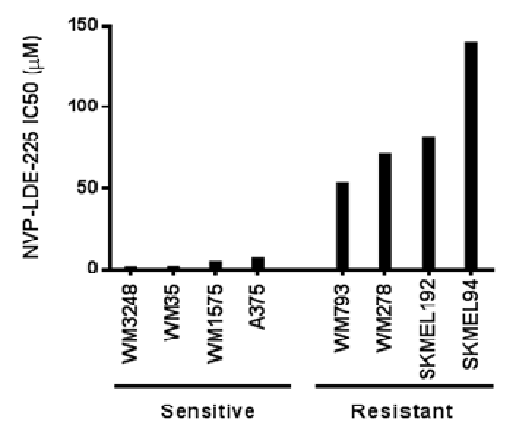

$C$

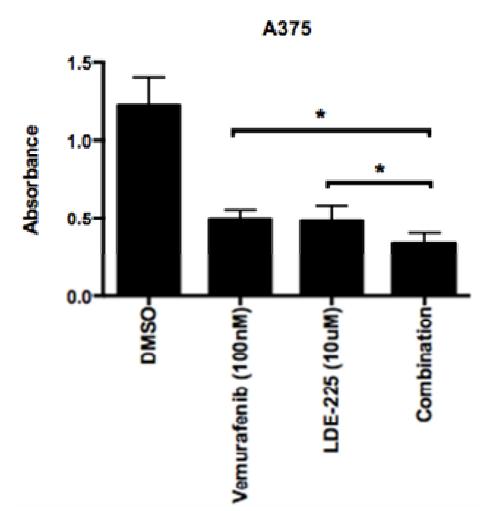

B
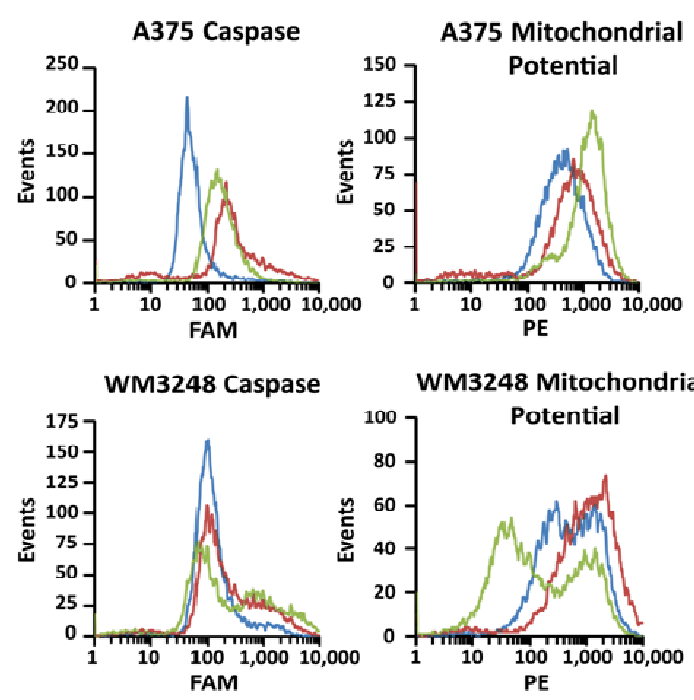

WM3248 Mitochondrial

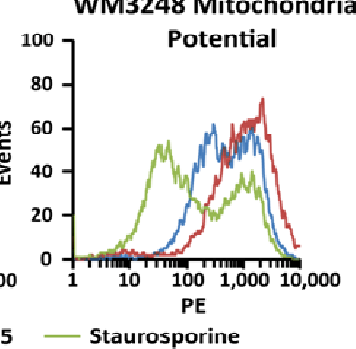

We then examined the impact of BRAF mutation status on the response (or lack thereof) to hedgehog pathway inhibition. Our data revealed that $36 \%(6 / 16)$ of the V600E B-Raf mutant melanoma cell lines had modest expression of GLI2 and SMO as compared to melanocytes (Figure 2a,b). In our panel of V600E B-Raf mutant cell lines treated with NVP-LDE-225, we found that 36\% (4/11: WM35, WM3248, WM1575, and A375) were sensitive to the inhibitor $\left(\mathrm{IC}_{50}<10 \mu \mathrm{M}\right)$ (Figure 6). $50 \%(2 / 4)$ of the N-Ras mutant cell lines (WM1366 and WM1361A) were sensitive to the NVP-LDE-225, while none of the three B-Raf wild type cell lines (SkMel 173, 187, and 197) were sensitive. Likewise, the c-kit mutant cell line WM3211 and the two V599K B-Raf mutant cell lines SkMel 94 and SkMel 100 were resistant to the inhibitor $\left(\mathrm{IC}_{50}>50 \mu \mathrm{M}\right.$, Figure 6). The four BRAF mutant cell lines sensitive 
to NVP-LDE-225 demonstrated moderate GLI2 and SMO expression, as did the sensitive N-Ras mutant cell line, WM1361A.

Figure 6. A panel of 18 melanoma cell lines demonstrates differential sensitivity to NVP-LDE-225. WM35, WM3248, WM1575, A375, WM1361, and WM1366 were sensitive to NVP-LDE-225 $\left(\mathrm{IC}_{50}<15 \mu \mathrm{M}\right)$. WM1552, SkMel147, WM115, and RPMI7951 demonstrated intermediate sensitivity to NVP-LDE-225 ( $\mathrm{IC}_{50} 20 \mu \mathrm{M}-30 \mu \mathrm{M}$ ), while the remaining 11 cell lines were resistant to the inhibitor $\left(\mathrm{IC}_{50}>50 \mu \mathrm{M}\right)$. The values represent the mean from a representative experiment performed three times with similar results.

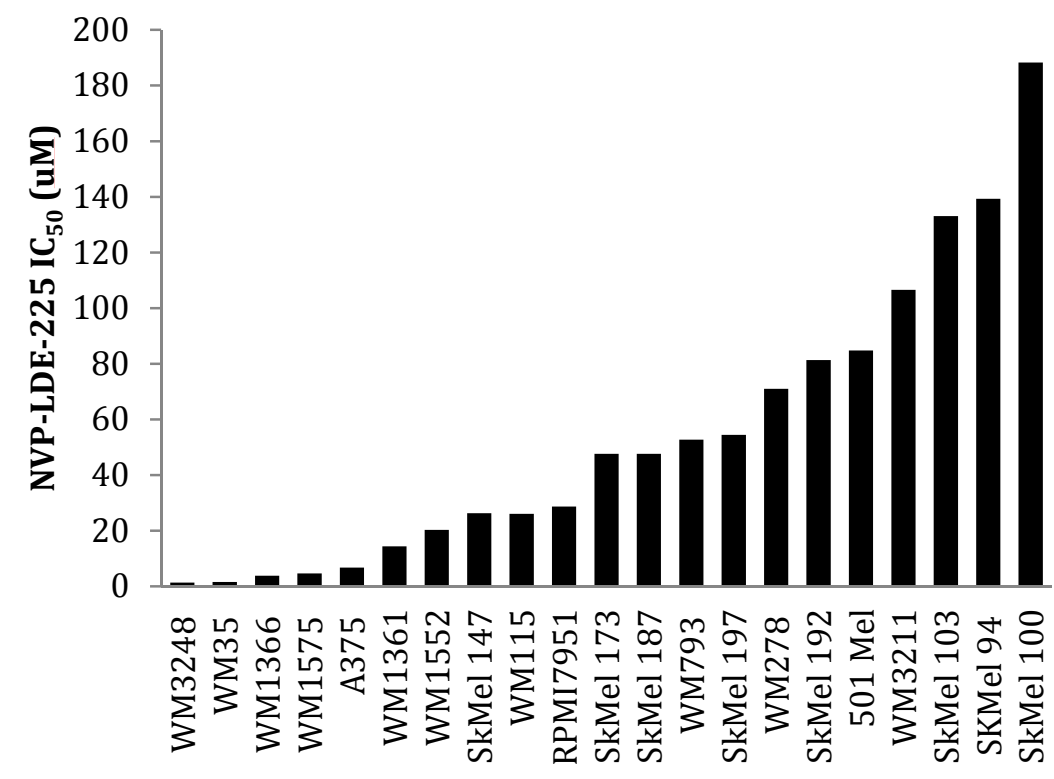

\subsection{NVP-LDE-225 Inhibits Melanoma Growth in Vivo}

As NVP-LDE-225 inhibited cell viability in vitro in a subset of our melanoma cell line panel, we wanted to determine whether oral administration of NVP-LDE-225 would inhibit melanoma growth in vivo. One week of daily $60 \mathrm{mg} / \mathrm{kg}$ NVP-LDE-225 suspension delivered by oral gavage to NOG mice bearing A375 xenografts resulted in decreased tumor volume $(p=0.103)$ and tumor weight $(p=0.0979)$ as compared to the vehicle treated mice (Figure $7 \mathrm{a}-\mathrm{c}$ ). The average mouse weight in the treatment group was lower than in the vehicle group; however the difference was not statistically significant (Figure 7c). The treatment duration was limited to one week as four of the ten mice in the treatment group exhibited direct local GI toxicity secondary to the suspension formulation used for oral gavage, an adverse event not observed in human phase I studies of NVP-LDE-225 capsules. RNA was extracted from vehicle and NVP-LDE-225 tumors and PTCH1 expression was found to be $60 \%$ lower in the NVP-LDE-225 treated tumor as compared to vehicle-treated tumors (Figure 7d), supporting effective targeting of the $\mathrm{HH}$ pathway. 
Figure 7. NVP- LDE-225 $60 \mathrm{mg} / \mathrm{kg}$ inhibits melanoma cell proliferation in vivo. (A) One week of daily NVP-LDE-225 $60 \mathrm{mg} / \mathrm{kg}$ administered by oral gavage to an A375 xenograft model in NOG mice inhibits melanoma growth better than vehicle. (B-C) Tumors in NVP-LDE-225 treated mice $(\mathrm{n}=6)$ are of lower volume $(p=0.103)$ and weight $(p=0.0979)$ than the tumors in vehicle treated mice $(n=7)$. The mice in the vehicle and LDE-225 treated groups are of similar weight throughout the duration of treatment $(p=0.691)$. (D) NVP-LDE-225 inhibits the hedgehog pathway as measured by decreased PTCH1 transcript in the tumors of NVP-LDE-225 treated mice as compared to the transcript levels in the tumors of control treated mice, as measured by qRT-PCR $(*, p<0.003)$. In $\mathrm{B}-\mathrm{C}$, values represent the mean \pm standard deviation of at least 6 animals per treatment group. In $\mathrm{D}$, the values represent the mean \pm standard deviation of triplicate qPCRs from the tumors of three mice from each treatment group.

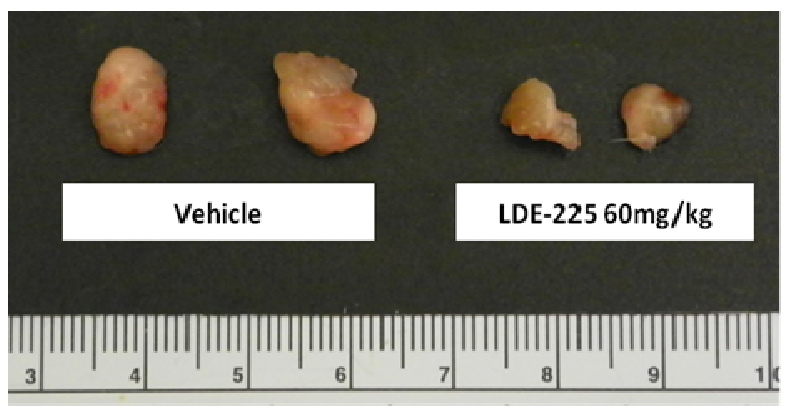

(A)
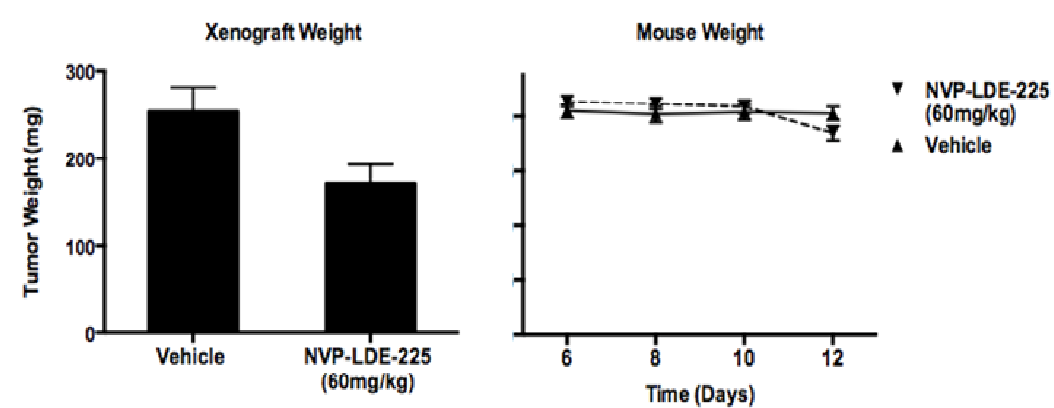

(C)

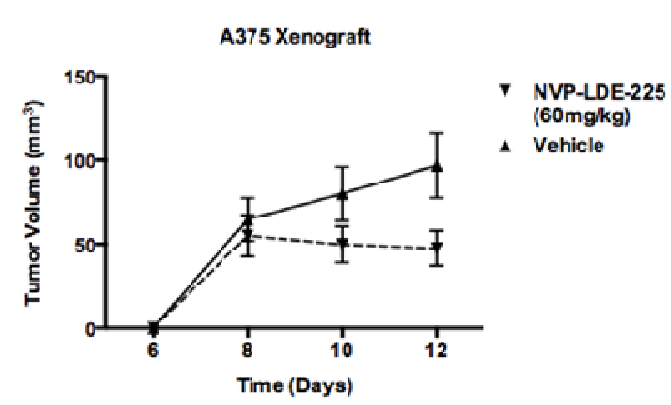

(B)

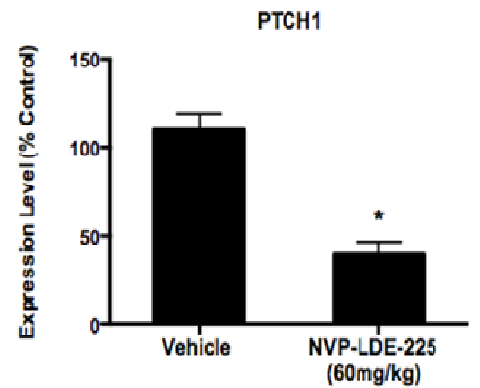

(D)

3.5. Increased SMO Levels are Associated with Decreased Survival, while Elevated GLI3 Levels are Associated with Better Survival in Metastatic Melanoma Patients

As prior reports describe increased expression of hedgehog pathway members in metastatic melanoma specimens as compared to primary melanoma tissue, we sought to examine if there was an association between hedgehog pathway activity and survival. Microarray gene expression profiles from 44 metastatic melanoma specimens were examined for expression levels of SHH, GLI1, GLI2, SMO, PTCH1, and WNT5 [29]. We then examined the association of these transcript levels with metastatic melanoma patient outcomes. In this cohort, elevated levels of SMO expression correlated with significantly decreased post-recurrence survival as compared to those patients expressing levels of SMO below the median expression value $(\mathrm{HR}=2.85, p=0.006)$. We isolated RNA from 30 additional metastatic melanoma specimens and performed U133 2.0 Plus Affymetrix array on these samples. Upon examining the pooled expression values from all 74 samples, there remained a 
statistically significant association between elevated SMO and decreased survival in the expanded cohort (Figure 8a, HR = 1.927, $p=0.0374$ ). SHH, GLI1, GLI2, PTCH1, and WNT5 were not associated with decreased survival in this expanded cohort. While the hedgehog pathway transcriptional repressor GLI3 was not detected by the U133A platform in any of our first 44 melanoma specimens, the U133 2.0 Plus array analysis did detect GLI3 transcript expression and revealed differential expression among the 30 metastatic melanoma specimens analyzed. Elevated expression of the hedgehog pathway transcriptional repressor GLI3 (as defined as above the median expression level) in the 30 metastatic tumor specimens associated with better survival in metastatic melanoma patients (Figure $8 \mathrm{~b}, \mathrm{HR}=0.15, p=0.015$ ). Microarray expression levels of GLI3 and SMO in patient tumors were confirmed by quantitative real time PCR (Figure $8 \mathrm{c}, \mathrm{d}$ ).

Figure 8. Hedgehog pathway activity is associated with decreased post-recurrence survival in metastatic melanoma patients. (A) Elevated SMO expression is associated with significantly shorter survival in metastatic melanoma patients (Hazard Ratio $=1.927$, $p=0.0374$ ). (B) Elevated GLI3 levels, a hedgehog pathway transcriptional repressor, is associated with increased survival in metastatic melanoma patients (Hazard Ratio $=0.15$, $p=0.015)$. (C-D) Transcript levels of SMO and GLI3 in metastatic melanoma specimens are verified using qRT- PCR. Patient specimen numbers are listed on the $\mathrm{x}$ axis. Low and high expression levels of SMO and GLI3 were defined as below or above the median expression value, $(*, p<0.05)$.

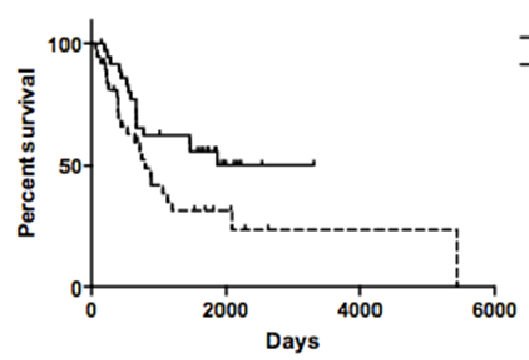

(A)

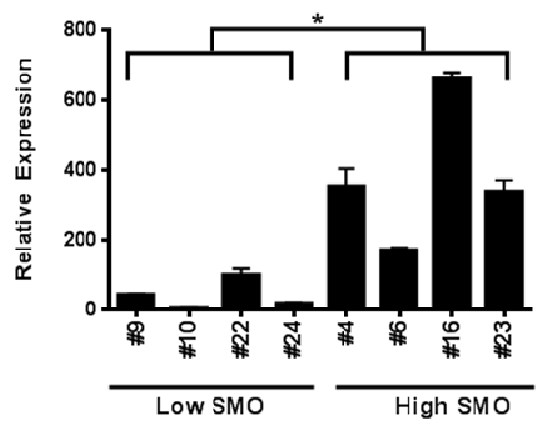

(C)
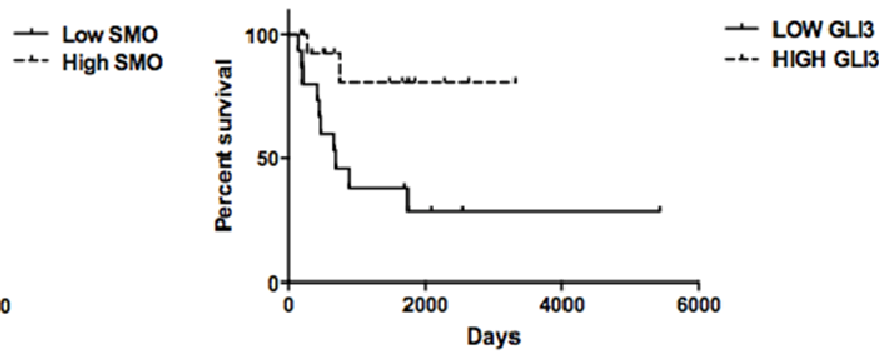

(B)

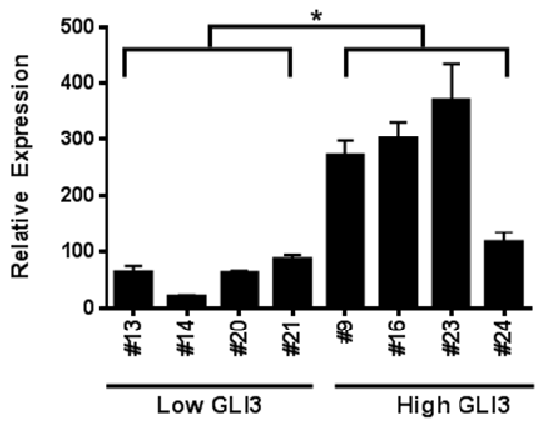

(D)

\subsection{SMO Inhibition Induces a Programmed Cell Death Gene Expression Signature and Downregulates} Embryonic Stem Cell Pluripotency

To determine the mechanism of SMO inhibitor-induced apoptosis, we performed gene expression profiling of melanoma cells treated with NVP-LDE-225 and SMO siRNA treated melanoma cells. Microarray expression profiles of WM3248 and A375 cell lines treated with siRNA to SMO or the 
small molecule NVP-LDE-225 were compared to non-targeting siRNA or DMSO treated controls, respectively. In the WM3248 cell line, both genetic interference and NVP-LDE-225 treatment for $24 \mathrm{~h}$ led to downregulation of cell adhesion molecules claudin20 and ICAM2, as well as the metalloproteases BMP1 and MMP28, while mediators of programmed cell death such as caspase I, forkhead BoxO3 pseudogene, FOS-like antigen 2, and BCL2 interacting protein 3 were upregulated $(p<0.05)$. In the A375 cell line, both siRNA to SMO and NVP-LDE-225 downregulated the growth factor PDGF-alpha and the protein translation mediator eIF4a2, while upregulating the apoptosis mediator annexin A9 and the melanoma antigen MAGE-E1 $(p<0.05)$. Interestingly, in both the A375 and WM3248 cell lines, NVP-LDE-225 downregulated the Ras signaling adaptor GRAP (-1.22 averaged fold change, $p<0.05)$ and upregulated XAF1 (+1.2 averaged fold change, $p<0.05)$, a mediator of programmed cell death. In both cell lines tested, the notch ligand DLL3 $(+1.23$ averaged fold change, $p=0.041$ ) and the WNT5a receptor RORA were upregulated by NVP-LDE-225 treatment $(+1.34$ averaged fold change, $p=0.0004)$.

Figure 9. Ingenuity Canonical Pathway analysis reveals downregulation of stem cell pluripotency signaling and cell cycle regulators. (A-D) Downregulated IPA (Ingenuity Pathway Analysis) canonical pathways are shown for A375 and WM3248 cell lines treated with either $5 \mu \mathrm{M}$ NVP-LDE-225 or SMO siRNA. The transcriptional profiles of the DMSO treated and non-targeting siRNA treated A375 and WM3248 cells were used as controls in the IPA analysis. The stem cell pathways that were downregulated by both SMO siRNA and NVP-LDE-225 in both cell lines are marked with an asterisk (*).

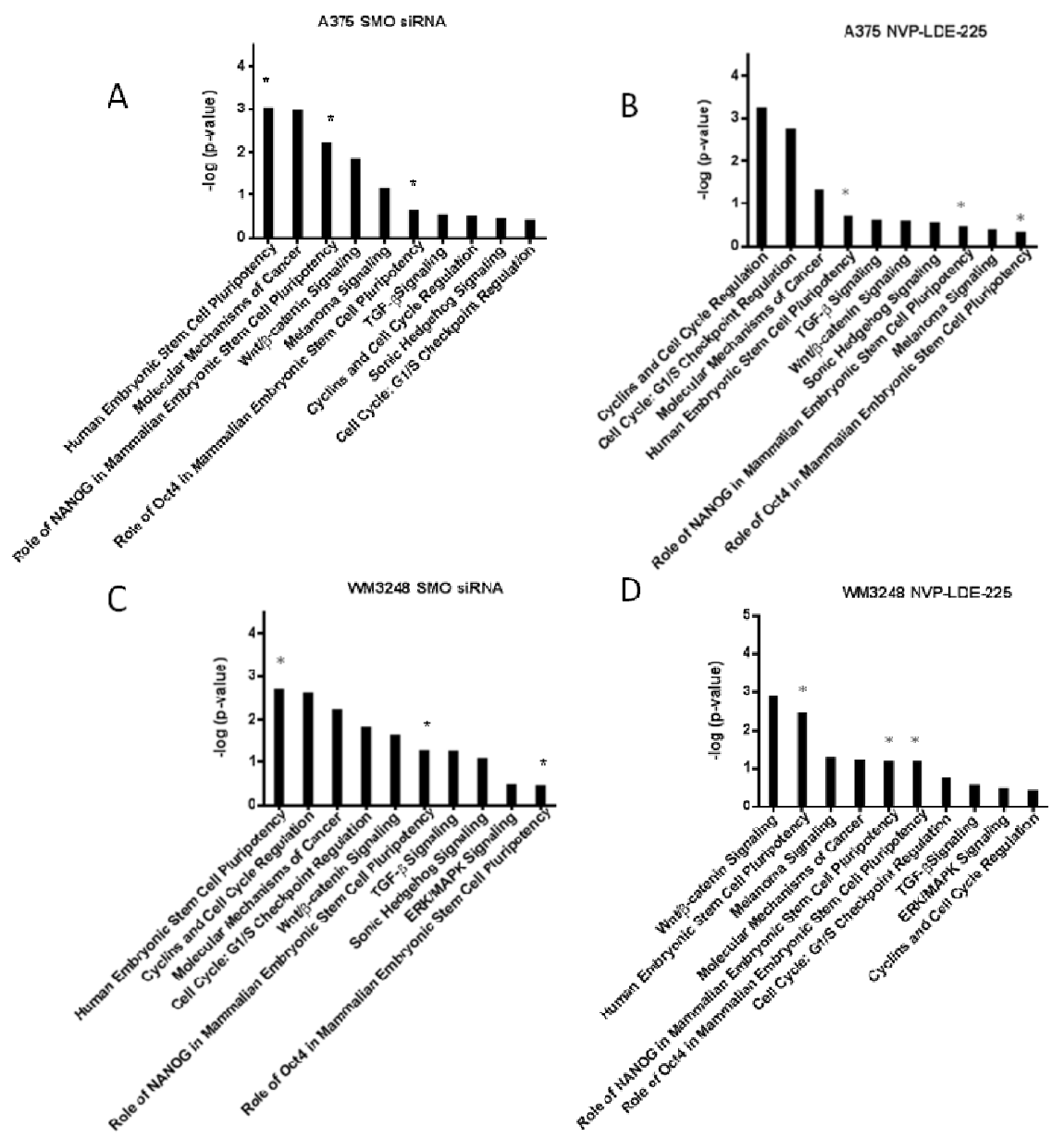


Ingenuity Pathway Analysis (IPA) was also performed on the transcriptional profile of NVP-LDE-225 and SMO siRNA treated melanoma cells. The pathways from the IPA canonical pathway library that were significantly downregulated by both SMO siRNA and NVP-LDE-225 in both the A375 and WM3248 cell lines are pictured in Figure 9. The IPA revealed downregulation of the Human Embryonic Stem Cell Pluripotency pathway in both SMO siRNA and NVP-LDE-225 treated WM3248 and A375 cells $(p<0.05)$. The role of NANOG in Mammalian Embryonic Stem Cell Pluripotency pathway and the role of Oct4 in Mammalian Embryonic Stem Cell Pluripotency pathway were also significantly downregulated in both SMO siRNA and inhibitor treated A375 and WM3248 cells $(p<0.05)$.

A heatmap of differentially expressed genes from the IPA human stem cell pluripotency pathway in SMO siRNA and NVP-LDE-225- treated cells as compared to their respective controls is pictured in Figure 10a,b. Our IPA analysis also showed that various apoptosis pathways were upregulated by SMO inhibition (Table 1). SMO siRNA upregulated the Death Receptor Signaling Pathway and MYC Mediated Apoptosis Signaling $(p<0.05)$. NVP-LDE-225 upregulated the Death Receptor Signaling Pathway, MYC Mediated Apoptosis Signaling, and Apoptosis Signaling in melanoma cell lines $(p<0.05)$.

Figure 10. Genetic and Pharmacologic Inhibition of SMO downregulates mediators of stem cell pluripotency. (A) SMO siRNA downregulates expression of IPA human stem cell pluripotency pathway members as compared to non-targeting (NT) siRNA treated WM3248 and A375 cells. (B) NVP-LDE-225 downregulates expression of IPA human stem cell pluripotency pathway members as compared to DMSO- treated WM3248 and A375 cells.

A

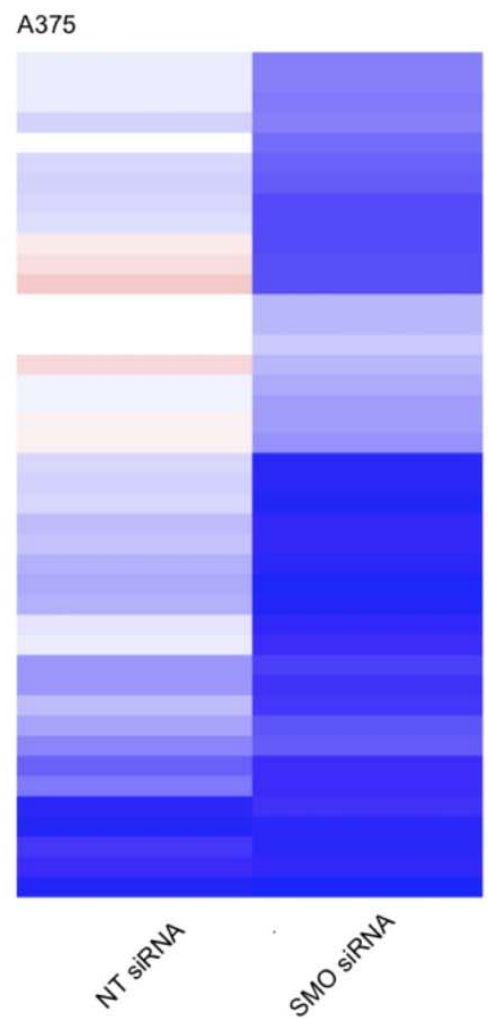

WM3248
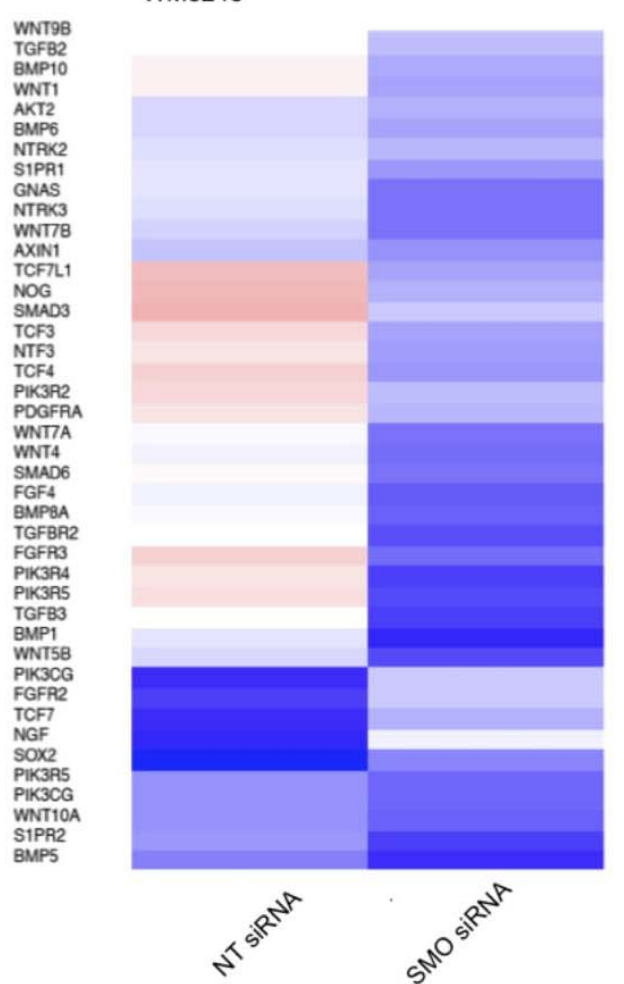

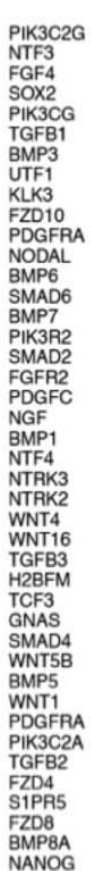

NANOG 
Figure 10. Cont.
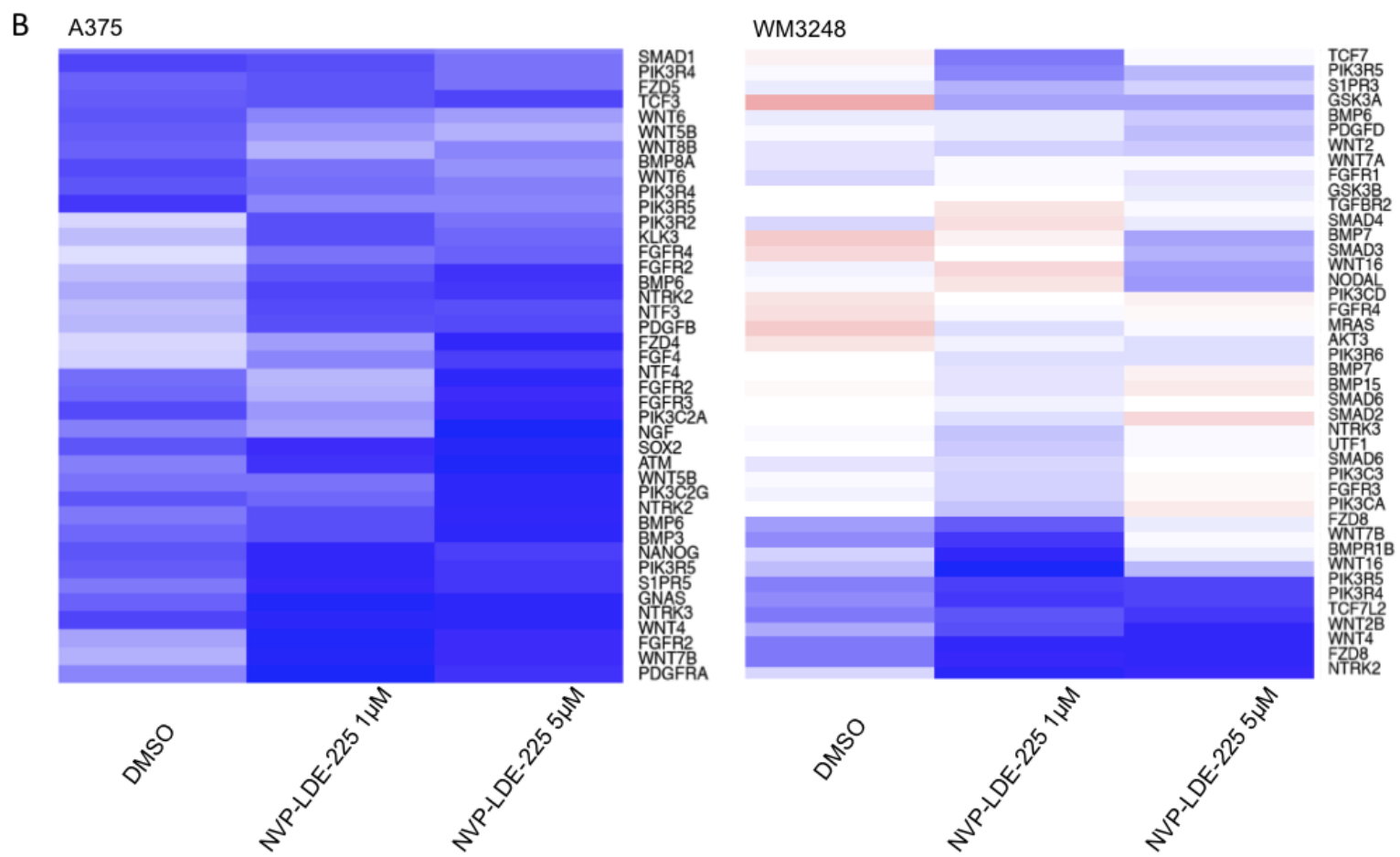

Table 1. Genetic and Pharmacologic Inhibition of SMO upregulates apoptosis signaling. Both SMO siRNA and NVP-LDE-225 upregulate apoptosis pathways in WM3248 and A375 cell lines.

\begin{tabular}{ccc}
\hline & A375 & \multicolumn{1}{c}{ WM3248 } \\
\hline SMO siRNA & -Death Receptor Signaling Pathway & -Myc Mediated Apoptosis Signaling \\
NVP-LDE-225 & -Apoptosis Signaling & -Death Receptor Signaling Pathway \\
& -Myc Mediated Apoptosis Signaling & -Myc Mediated Apoptosis Signaling \\
\hline
\end{tabular}

\section{Conclusions}

Here we show, for the first time, that elevated levels of hedgehog pathway components correlate with worse survival in melanoma patients. We also demonstrate that NVP-LDE-225, a small molecule SMO inhibitor already in clinical trials in other solid tumors, inhibits the hedgehog pathway effectively and has antitumor activity against B-RAF mutant melanoma both in vitro and in vivo. Our findings that NVP-LDE-225 inhibits proliferation and induces apoptosis in melanoma cell lines agree with those of Jalili et al. However, in contrast to their study, we observed a more dramatic antiproliferative effect in B-RAF mutant cell lines as compared to B-RAF wild type cells and we did not observe a cell cycle arrest upon NVP-LDE-225 treatment. The relative resistance of B-RAF wild type melanoma cell lines and the lack of cell cycle arrest observed in our study may be secondary to the different melanoma cell lines used. While Jalili et al. observed cell cycle arrest in NVP-LDE-225 treated UACC 257 and LOX IMVI cell lines, we observed no cell cycle alterations in NVP-LDE-225 treated WM3248 and A375 melanoma cell lines. Similarly, while Jalili et al. demonstrated sensitivity of MEWO and MEL FH wild type B-RAF cell lines to NVP-LDE-225, the three wild type B-RAF cell lines (SkMel173, SkMe1187, and SkMe1197) used in our study were resistant to NVP-LDE-225, with $\mathrm{IC}_{50} \mathrm{~S}>50 \mu \mathrm{M}$. 
Finally, the more robust in vivo antitumor response observed in Jalili et al.'s xenograft model may be secondary to the direct intratumoral delivery of NVP-LDE-225 in their animal model as compared to the oral gavage delivery method used in our study.

Our observations suggest that hedgehog pathway inhibitors may be an additional therapeutic strategy against B-RAF mutant melanoma. As activated RAS/MAPK signaling has been shown to induce hedgehog pathway activity via translocation of GLI1 and GLI2 into the nucleus, it is possible that B-RAF mutation is augmenting hedgehog pathway activity in our melanoma cell lines and that inhibiting both B-RAF and the hedgehog pathway may result in an enhanced therapeutic effect. Our data demonstrate a modest but significant effect of combining B-RAF and hedgehog inhibitors, but additional studies of the dosage and sequence of administration in vivo are necessary to better define a clinically useful regimen. Whether SMO inhibition might be effective in vemurafenib resistant B-RAF mutant tumors is currently under investigation in our laboratory.

Although other groups have reported upregulation of hedgehog pathway members in metastatic tissue as compared to primary specimens, this is the first report of an association of SMO levels with significantly decreased survival in metastatic melanoma patients. The protective effect of elevated GLI3 levels underscores the role of hedgehog pathway activation in more aggressive disease. We also report that a clinically relevant inhibitor already in phase II clinical trials inhibits melanoma growth in vitro and in vivo. In ongoing clinical trials in advanced basal cell carcinoma, this daily dosed oral inhibitor has been well tolerated with little toxicity. Thus, our findings could be readily translated into clinical trials of a SMO inhibitor in combination with vemurafenib in patients whose B-RAF mutant tumors express GLI2 and SMO at moderate levels.

Ingenuity pathway analysis revealed that silencing of the hedgehog pathway by either chemical or genetic means significantly inhibited the expression of human embryonic stem cell pluripotency related genes. This result indicates that SMO inhibitors may effectively target the melanoma stem cell compartment and supports a recent study showing that melanoma-initiating cells require hedgehog signaling in order to self-renew [25]. In order to achieve a durable antitumor response, it may be necessary to not only target mutated oncogenic pathways in melanoma (i.e., B-RAF), but also the embryonic signaling pathways promoting self renewal in a subset of melanoma cells. Inhibition of melanoma initiating cell self-renewal might extend the durability of the robust, albeit short-lived, antitumor responses achieved with vemurafenib in metastatic melanoma patients.

NVP-LDE-225 induced a programmed cell death gene expression profile in both cell lines tested, and also paradoxically upregulated the notch ligand DLL3 and the WNT5 receptor ROR in both the A375 and WM3248 cell lines. As both of the cell lines tested demonstrated upregulation of the programmed cell death mediator XAF1 in response to both small molecule and genetic SMO inhibition, this protein is likely a key mediator in hedgehog inhibitor-induced apoptosis. The upregulation of the notch ligand DLL3 and WNT5 that we observed in our microarray analysis indicate a compensatory upregulation of alternative stem cell signaling pathways in response to SMO inhibition. Compensatory upregulation of alternate stem cell pathways is a phenomenon previously reported with Notch inhibitors in neural tumors [30]. Schreck et al demonstrated that single agent Notch inhibition activated hedgehog signaling, which induced resistance to Notch inhibition, suggesting a dynamic crosstalk between the Hedgehog and Notch pathways. Combined, simultaneous targeting of Notch and Hedgehog pathways overcame the resistance to single agent-mediated Notch 
inhibition in glioblastoma and medulloblastoma models [30]. Hedgehog signaling has been shown to be necessary for the maintenance of glioblastoma and melanoma-initiating cells. Likewise, Notch signaling was found to be necessary for the maintenance of melanoma-initiating cells [10]. In light of these findings, our data suggest that combined blockade of the Hedgehog and Notch pathways, or perhaps WNT5 signaling, may be the optimal strategy for preventing the self-renewal of melanoma initiating cells.

Furthermore, we identified overexpression of GLI2 and SMO as a signature of resistance to smoothened inhibition in melanoma, as has been reported recently in human medulloblastoma using both NVP-LDE-225 and Vismodegib, an oral SMO inhibitor that is FDA-approved in advanced basal cell carcinoma. Two studies have reported amplifications of chromosomal regions containing GLI2 in Vismodegib and NVP-LDE-225-resistant medulloblastoma cell lines [31-33]. Our findings in the WM278 cell line suggest that, in melanoma harboring amplification of GLI2, hedgehog target genes are constitutively transcribed and upstream inhibition of hedgehog signaling via SMO inhibitors is ineffective. Our results indicate, that in those melanoma cells harboring GLI2 amplification, direct targeting of GLI2 is the best antitumor strategy. Pre-clinical evaluation of GLI-specific inhibitors is currently underway [34-36].

As NVP-LDE-225 enhanced the cytotoxic effects of single agent B-RAF inhibition, SMO inhibition may be effective in combination with vemurafenib in patients whose tumors express moderate levels of GLI2 and SMO. Based on the responses to NVP-LDE-225 in our melanoma cell line panel, it appears that a subset of patients whose tumors harbor either V600E B-RAF mutations or N-RAS mutation may benefit most from inhibition of SMO.

In summary, our pre-clinical studies support SMO as a therapeutic target in melanoma and offer insights into the clinical potential of this new treatment strategy both as a single agent and in combination with other targeted agents in metastatic melanoma patients.

\section{Acknowledgments}

We thank the NYU Genome Technology Center (supported in part by NIH/NCI P30 CA016087-30 grant) for expert assistance with microarray experiments.

\section{Conflicts of Interest}

The authors declare no conflict of interest.

\section{References}

1. National Cancer Institute. SEER Stat Fact Sheets: Melanoma of the Skin. Available online: $\mathrm{http} / / /$ seer.cancer.gov/statfacts/html/melan.html/ (accessed on 29 January 2013).

2. Garbe, C.; McLeod, G.R.; Buettner, P.G. Time trends of cutaneous melanoma in Queensland, Australia and Central Europe. Cancer 2000, 89, 1269-1278.

3. Howlader, N.; Noone, A.M.; Krapcho, M.; Garshell, J.; Neyman, N.; Altekruse, S.F.; Kosary, C.L.; Yu, M.; Ruhl, J.; Tatalovich, Z.; et al. SEER Cancer Statistics Review, 1975-2009 (Vintage 2009 Populations). Available online: http://seer.cancer.gov/csr/1975_2009_pops09/ (accessed on 31 October 2003). 
4. Bedikian, A.Y.; Millward, M.; Pehamberger, H.; Conry, R.; Gore, M.; Trefzer, U.; Pavlick, A.C.; de Conti, R.; Hersh, E.M.; Hersey, P.; et al. Bcl-2 antisense (oblimersen sodium) plus dacarbazine in patients with advanced melanoma: The Oblimersen Melanoma Study Group. J. Clin. Oncol. 2006, 24, 4738-4345.

5. Chapman, P.B.; Hauschild, A.; Robert, C.; Haanen, J.B.; Ascierto, P.; Larkin, J.; Dummer, R.; Garbe, C.; Testori, A.; Maio, M.; et al. Improved survival with vemurafenib in melanoma with BRAF V600E mutation. N. Engl. J. Med. 2011, 364, 2507-2516.

6. Solit, D.; Sawyers, C.L. Drug discovery: How melanomas bypass new therapy. Nature 2010, 468, 902-903.

7. Hodi, F.S.; O’Day, S.J.; McDermott, D.F.; Weber, R.W.; Sosman, J.A.; Haanen, J.B.; Gonzalez, R.; Robert, C.; Schadendorf, D.; Hassel, J.C.; et al. Improved survival with ipilimumab in patients with metastatic melanoma. N. Engl. J. Med. 2010, 363, 711-723.

8. Robert, C.; Thomas, L.; Bondarenko, I.; O’Day, S.; Weber, J.; Garbe, C.; Lebbe, C.; Baurain, J.F.; Testori, A.; Grob, J.J.; et al. Ipilimumab plus dacarbazine for previously untreated metastatic melanoma. N. Engl. J. Med. 2011, 364, 2517-2526.

9. Zhang, K.; Wong, P.; Zhang, L.; Jacobs, B.; Borden, E.C.; Aster, J.C.; Bedogni, B. A Notch1-neuregulin1 autocrine signaling loop contributes to melanoma growth. Oncogene 2012, 31, 4609-4618.

10. Huynh, C.; Poliseno, L.; Segura, M.F.; Medicherla, R.; Haimovic, A.; Menendez, S.; Shang, S.; Pavlick, A.; Shao, Y.; Darvishian, F.; et al. The novel gamma secretase inhibitor R04929097 reduces the tumor initiating potential of melanoma. PLoS One 2011, 6, e25264.

11. Tolcher, A.W.; Messersmith, W.A.; Mikulski, S.M.; Papadopoulos, K.P.; Kwak, E.L.; Gibbon, D.G.; Patnaik, A.; Falchook, G.S.; Dasari, A.; Shapiro, G.I.; et al. Phase I study of R04929097, a gamma secretase inhibitor of Notch signaling, in patients with refractory metastatic or locally advanced solid tumors. J. Clin. Oncol. 2012, 30, 2348-2353.

12. Imbimbo, B.P. Therapeutic potential of gamma-secretase inhibitors and modulators. Curr. Top. Med. Chem. 2008, 8, 54-61.

13. Okamoto, R.; Tsuchiya, K.; Nemoto, Y.; Akiyama, J.; Nakamura, T.; Kanai, T.; Watanabe, M. Requirement of Notch activation during regeneration of the intestinal epithelia. Am. J. Physiol. Gastrointest. Liver Physiol. 2009, 296, G23-G35.

14. Stecca, B.; Mas, C.; Clement, V.; Zbinden, M.; Correa, R.; Piguet, V.; Beermann, F.; Ruiz, I.; Altaba, A. Melanomas require HEDGEHOG-GLI signaling regulated by interactions between GLI1 and the RAS-MEK/AKT pathways. Proc. Natl. Acad. Sci. USA 2007, 104, 5895-900.

15. Alexaki, V.I.; Javelaud, D.; van Kempen, L.C.; Mohammad, K.S.; Dennler, S.; Luciani, F.; Hoek, K.S.; Juàrez, P.; Goydos, J.S.; Fournier, P.J.; et al. GLI2-mediated melanoma invasion and metastasis. J. Natl. Cancer Inst. 2010, 102, 1148-1159.

16. Das, S.; Harris, L.G.; Metge, B.J.; Liu, S.; Riker, A.I.; Samant, R.S.; Shevde, L.A. The hedgehog pathway transcription factor GLI1 promotes malignant behavior of cancer cells by up-regulating osteopontin. J. Biol. Chem. 2009, 284, 22888-22897.

17. Rubin, L.L.; de Sauvage, F.J. Targeting the hedgehog pathway in cancer. Nat. Rev. Drug Discov. 2006, 5, 1026-1033. 
18. Thayer, S.P.; di Magliano, M.P.; Heiser, P.W.; Nielsen, C.M.; Roberts, D.J.; Lauwers, G.Y.; Qi, Y.P.; Gysin, S.; Fernández-del Castillo, C.; Yajnik, V.; et al. Hedgehog is an early and late mediator of pancreatic cancer tumorigenesis. Nature 2003, 425, 851-856.

19. Kubo, M.; Nakamura, M.; Tasaki, A.; Yamanaka, N.; Nakashima, H.; Nomura, M.; Kuroki, S.; Katano, M. Hedgehog signaling pathway is a new therapeutic target for patients with breast cancer. Cancer Res. 2004, 64, 6071-6074.

20. Berman, D.M.; Karhadkar, S.S.; Maitra, A.; Montes de Oca, R.; Gerstenblith, M.R.; Briggs, K.; Parker, A.R.; Shimada, Y.; Eshleman, J.R.; Watkins, D.N.; et al. Widespread requirement for hedgehog ligand stimulation in growth of digestive tract tumours. Nature 2003, 425, 846-851.

21. Watkins, D.N.; Berman, D.M.; Burkholder, S.G.; Wang, B.; Beachy, P.A.; Baylin, S.B. Hedgehog signalling within airway epithelial progenitors and in small-cell lung cancer. Nature 2003, 422, 313-317.

22. Berman, D.M.; Karhadkar, S.S.; Hallahan, A.R.; Pritchard, J.I.; Eberhart, C.G.; Watkins, D.N.; Chen, J.K.; Cooper, M.K.; Taipale, J.; Olson, J.M.; et al. Medulloblastoma growth inhibition by hedgehog pathway blockade. Science 2002, 297, 1559-1561.

23. Merchant, A.A.; Matsui, W. Targeting hedgehog-A cancer stem cell pathway. Clin. Cancer Res. 2010, 16, 3130-3140.

24. Takebe, N.; Warren, R.Q.; Ivy, S.P. Breast cancer growth and metastasis: interplay between cancer stem cells, embryonic signaling pathways and epithelial-to-mesenchymal transition. Breast Cancer Res. 2011, 13, 211.

25. Santini, R.; Vinci, M.C.; Pandolfi, S.; Penachioni, J.Y.; Montagnani, V.; Olivito, B.; Gattai, R.; Pimpinelli, N.; Gerlini, G.; Borgognoni, L.; et al. Hedgehog-GLI signaling drives self-renewal and tumorigenicity of human melanoma-initiating cells. Stem Cells 2012, 30, 1808-1818.

26. Jalili, A.; Mertz, K.D.; Romanov, J.; Wagner, C.; Kalthoff, F.; Stuetz, A.; Pathria, G.; Gschaider, M.; Stingl, G.; Wagner, S.N. NVP-LDE225, a potent and selective SMOOTHENED antagonist reduces melanoma growth in vitro and in vivo. PLoS One 2013, 8, e69064.

27. Fang, D.; Nguyen, T.K.; Leishear, K.; Finko, R.; Kulp, A.N.; Hotz, S.; Van Belle, P.A.; Xu, X.; Elder, D.E.; Herlyn, M. A tumorigenic subpopulation with stem cell properties in melanomas. Cancer Res. 2005, 65, 9328-9337.

28. Rose, A.E.; Poliseno, L.; Wang, J.; Clark, M.; Pearlman, A.; Wang, G.; de Miera, E.C.; Medicherla, R.; Christos, P.J.; Shapiro, R.; et al. Integrative genomics identifies molecular alterations that challenge the linear model of melanoma progression. Cancer Res. 2011, 71, 2561-2571.

29. Bogunovic, D.; O’Neill, D.W.; Belitskaya-Levy, I.; Vacic, V.; Yu, Y.L.; Adams, S.; Darvishian, F.; Berman, R.; Shapiro, R.; Pavlick, A.C.; et al. Immune profile and mitotic index of metastatic melanoma lesions enhance clinical staging in predicting patient survival. Proc. Natl. Acad. Sci. USA 2009, 106, 20429-20434.

30. Schreck, K.C.; Taylor, P.; Marchionni, L.; Gopalakrishnan, V.; Bar, E.E.; Gaiano, N.; Eberhart, C.G. The Notch target Hes1 directly modulates Glil expression and Hedgehog signaling: a potential mechanism of therapeutic resistance. Clin. Cancer Res. 2010, 16, 6060-6670. 
31. Dijkgraaf, G.J.; Alicke, B.; Weinmann, L.; Januario, T.; West, K.; Modrusan, Z.; Burdick, D.; Goldsmith, R.; Robarge, K.; Sutherlin, D.; et al. Small molecule inhibition of GDC-0449 refractory smoothened mutants and downstream mechanisms of drug resistance. Cancer Res. 2011, 71, 435-444.

32. Buonamici, S.; Williams, J.; Morrissey, M.; Wang, A.; Guo, R.; Vattay, A.; Hsiao, K.; Yuan, J.; Green, J.; Ospina, B.; et al. Interfering with resistance to smoothened antagonists by inhibition of the PI3K pathway in medulloblastoma. Sci. Transl. Med. 2010, 2, 51 ra70.

33. Metcalfe, C.; de Sauvage, F.J. Hedgehog fights back: Mechanisms of acquired resistance against Smoothened antagonists. Cancer Res. 2011, 71, 5057-5061.

34. Li, C.; Chi, S.; Xie, J. Hedgehog signaling in skin cancers. Cell. Signal. 2011, 23, 1235-1243.

35. Mas, C.; Ruiz, I.; Altaba, A. Small molecule modulation of HH-GLI signaling: current leads, trials and tribulations. Biochem. Pharmacol. 2010, 80, 712-723.

36. Takebe, N.; Harris, P.J.; Warren, R.Q.; Ivy, S.P. Targeting cancer stem cells by inhibiting Wnt, Notch, and Hedgehog pathways. Nat. Rev. Clin. Oncol. 2011, 8, 97-106.

(C) 2013 by the authors; licensee MDPI, Basel, Switzerland. This article is an open access article distributed under the terms and conditions of the Creative Commons Attribution license (http://creativecommons.org/licenses/by/3.0/). 